\title{
Initial evaluation of congenital hypothyroidism: a survey of general paediatricians in East Anglia
}

\author{
S F Ahmed, N D Barnes, I A Hughes
}

\begin{abstract}
The practice of general paediatricians in the initial evaluation of congenital hypothyroidism (CHT) was assessed. This was performed by a questionnaire survey of paediatricians in East Anglia of whom $84 \%$ responded. Nineteen of 25 clinicians based in seven district hospitals managed children with CHT. The median number of children in the care of each clinician was 4 (range 1-17) and the median number of children attending each hospital was 12 (range 5-23). All except one clinician arranged to confirm the diagnosis with a serum thyroid stimulating hormone concentration and free or total thyroxine. There was variation of opinion on the value of serum triiodothyronine and free triiodothyronine measurements, antibody screening, knee radiographs, and thyroid isotope scans. One clinician sought advice when notified of new children and two expressed the need for an investigation protocol. These findings indicate that most general paediatricians in East Anglia manage only a few children with CHT; the initial diagnosis is appropriately confirmed but they are uncertain about the value of other investigations. (Arch Dis Child 1997;77:339-341)
\end{abstract}

Keywords: hypothyroidism; investigation; screening

Congenital hypothyroidism (CHT) affects approximately one in 3500 to 4000 babies. In the UK the diagnosis is now made in nearly all cases by biochemical screening of the newborn. These programmes began in the mid-1970s and the first regional programme to be established was in East Anglia. ${ }^{1}$ The various health regions in the UK have developed their own policies on notification procedures. Children with CHT who live in areas without a specialist paediatric endocrinology service are usually investigated and managed by general paediatricians. The purpose of the initial evaluation of a neonate identified by the screening laboratory is, firstly, to confirm the diagnosis by formal thyroid function tests; secondly, to start thyroxine replacement in appropriate dosage as promptly as possible; and, thirdly, to inform the family about the condition, its management, and prognosis. As there are no national guidelines on the initial evaluation and investigation of CHT we have surveyed the present practice of general paediatricians in East Anglia.

\section{Methods}

Details of all cases of CHT were obtained from the regional neonatal biochemical screening laboratory at Peterborough District Hospital. A list of investigations that may be relevant to investigation of suspected CHT was sent to all general paediatricians in the region (see table 1). Clinicians were asked to specify which tests they would perform at the initial evaluation, whether they felt any of the tests were unnecessary, and if they were uncertain of the value of any of the tests.

\section{Results}

Among 25 paediatricians based in seven district general hospitals, 19 managed children with CHT. A paediatric endocrinologist (NDB) held a specialist endocrine clinic at regular intervals at five of the seven hospitals. The questionnaire response rate was $84 \%$. The median number of cases managed by each clinician at the time of the survey was 4 (range 1-17) and the median number of cases per hospital was 12 (range 5-23). For the three paediatricians who did not respond, the figures were based on information obtained from the regional screening centre.

Figure 1 shows the results of the survey in respect of the investigations performed at the initial evaluation. All but one clinician confirmed the diagnosis with a serum thyroid

Table 1 List of investigations on which the general paediatricians were asked to comment: 'I would perform', 'I would perform if facilities', 'Not necessary', 'No views'

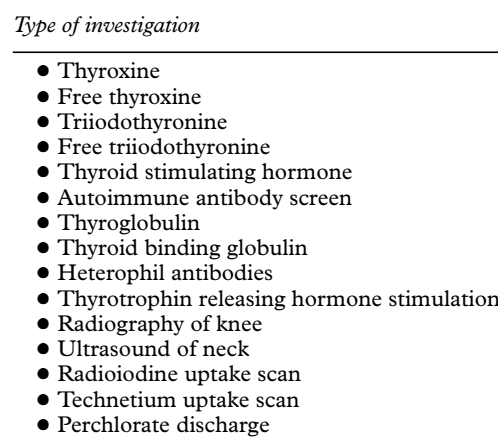




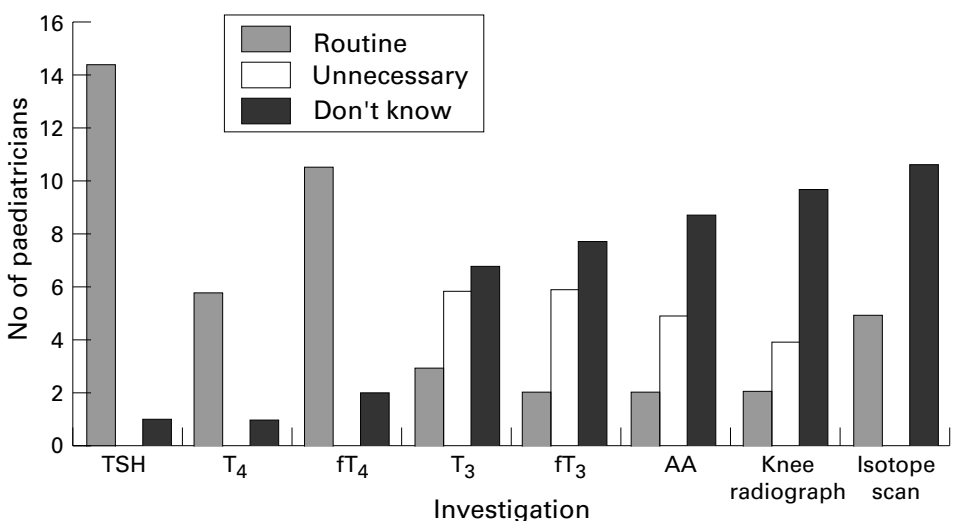

Figure 1 Response of paediatricians towards some of the commoner investigations performed at the initial evaluation of congenital hypothyroidism (isotope scans were performed at the age of 2 years). $T S H=$ thyroid stimulating hormone; $T_{4}=$ thyroxine; $f T_{4}$ = free thyroxine; $T_{3}=$ triiodothyronine; $T_{3}=$ free triiodothyronine; $A A=$ autoantibodies.

stimulating hormone and thyroxine/free thyroxine estimation. There was considerable variation of opinion with respect to the value of serum triiodothyronine and free triiodothyronine measurements, antibody screening, knee radiographs, and thyroid isotope scans. Clinicians performing an isotope scan did so as part of a re-evaluation, off treatment, at the age of 2 years. The majority of paediatricians were uncertain about the value of the other investigations listed on the questionnaire, one routinely sought expert advice when notified of a new case of CHT and two spontaneously expressed the need for an investigation protocol.

\section{Discussion}

CHT is one of the commonest congenital disorders that needs lifelong treatment. Opinions among paediatric endocrinologists differ over whether every case of CHT should be seen by a specialist but some experience in paediatric endocrinology is felt to be necessary. ${ }^{2}$ Recent studies show that the long term neurodevelopmental outcome of children on apparently

Local hospital $\quad$ Confirmed high TSH and low $\mathrm{T}_{4} \longrightarrow$ Start $\mathrm{T}_{4}$

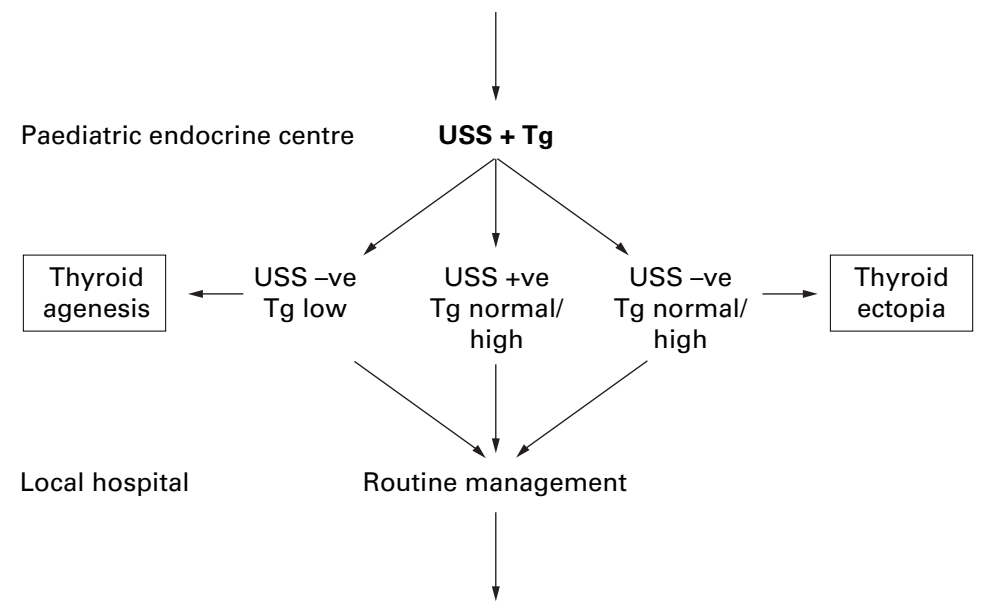

Paediatric endocrine Re-evaluation at 2 years centre

Figure 2 Authors'suggested guidelines for initial evaluation of congenital hypothyroidism. $\mathrm{Tg}=$ thyroglobulin $; \mathrm{TSH}=$ thyroid stimulating hormone; $T_{4}=$ thyroxine $;$ USS $=$ ultrasound scan. adequate replacement therapy may not be optimal, ${ }^{3}$ but the reason is not clear. Raised serum thyroid stimulating hormone with adequate thyroxine concentrations are often encountered in infancy. Undertreatment or lack of compliance may play a part but this could also be due to a developmental variability in intrapituitary deiodination of thyroxine to triiodothyronine. ${ }^{4}$ If the condition remains somewhat mysterious to them, the child is well, and missed doses have no apparent ill effect, some parents may become careless about ensuring the child continues with replacement therapy. Thorough clinical and biochemical evaluation and occasionally molecular biology techniques may help in reaching an aetiological diagnosis. ${ }^{5}$ Better understanding of the pathophysiology of CHT may help to improve medical management and patient compliance.

Our survey of general paediatricians in the region where a screening programme was first established in the UK showed that in most hospitals children with CHT are under the care of more than one paediatrician. New cases are allocated to consultants depending on the on-call rota. This policy is in contrast to that adopted for most other lifelong disorders such as diabetes mellitus, when designated clinicians care for all cases in a district. ${ }^{6}$ Although the diagnosis of CHT was appropriately confirmed with thyroxine and thyroid stimulating hormone measurement, clinicians were not clear about the value of other investigations for evaluating CHT.

Guidelines issued by the European Society for Paediatric Endocrinology ${ }^{7}$ and the American Academy of Pediatrics ${ }^{8}$ do not clearly state which investigations, other than thyroxine and thyroid stimulating hormone, need to be performed in initial evaluation. Understanding of the aetiology of CHT will improve if clear guidelines are formulated for evaluating every new case of CHT. Regional policies exist in some parts of the UK and differences in practice probably reflect differences in opinion between specialists in the field. We think that the care of newly diagnosed children should be shared between the local paediatrician and a paediatric endocrinologist. Figure 2 represents a protocol devised as a result of this study but which needs to be further evaluated. After initial examination and confirmation of the diagnosis, treatment can be started at the local hospital with early referral for a paediatric endocrine opinion. At this stage some investigations that do not require cessation of treatment, such as thyroglobulin ${ }^{9}$ and an ultrasound scan of the neck, ${ }^{10}$ can be performed to help elucidate the aetiology and allow an informed discussion with parents. Unusual problems can be assessed and arrangements made for a further thorough evaluation at around 2 years. This would be performed at the paediatric endocrine centre, while routine management in between these two evaluations as well as after the second evaluation could be undertaken at the local hospital.

We thank Dr A Heeley for allowing access to case details at the East Anglia regional biochemical neonatal screening centre, 
Peterborough. We also thank all the paediatricians in East Anglia who helped with this survey.

1 Barnes ND. Screening for congenital hypothyroidism: the first decade. Arch Dis Child 1985;60:587-92.

2 Gruters A. Screening for congenital hypothyroidism: effectiveness and clinical outcome. In: Kelnar $\mathrm{CJH}$, ed. Balliere's Clinical Paediatrics 1996;4:259-76.

3 Simons WF, Fuggle PW, Grant DB, Smith I. Intellectual development at 10 years in early treated congenital hypothyroidism. Arch Dis Child 1994;71:232-4.

4 Leonard JL, Koehrle J. Intracellular pathways of iodothyronine metabolism. In: Braverman $\mathrm{LE}$, Utiger $\mathrm{RD}$, eds. Lippincott-Raven, 1996.

5 Gruters A, Finke R, Krude H, Meinhold H. Etiology group- ing of permanent congenital hypothyroidism with a thyroid gland in situ. Horm Res 1994;41:3-9.

6 Betts P, Buckley M, Davies R, McEvilly E, Swift P. The care of young people with diabetes. Diabet Med 1996;13:S54-9.

7 ESPE Working Group on Congenital Hypothyroidism. Guidelines for neonatal screening programs for congenital hypothyroidism. Horm Res 1994;41:1-2.

8 American Academy of Pediatrics. Newborn screening for congenital hypothyroidism: recommended guidelines. Pediatrics 1993;91:1203-9.

9 Mitchell ML, Hermos RJ. Measurement of thyroglobulin in newborn screening specimens from normal and hypothyroid infants. Clin Endocrinol (Oxf) 1995;42:523-7.

10 Takashima S, Nomura N, Tanaka H, Itoh Y, Miki K, Harada T. Congenital hypothyroidism: assessment with ultra-
sound. American fournal of Neuroradiology 1995;16:111723. 